\title{
Numerical Solution of Nonlinear Fractional Volterra Integro-Differential Equations via Bernoulli Polynomials
}

\author{
Emran Tohidi, ${ }^{1}$ M. M. Ezadkhah, ${ }^{2}$ and S. Shateyi ${ }^{3}$ \\ ${ }^{1}$ Department of Mathematics, Aligoudarz Branch, Islamic Azad University, Aligoudarz, Iran \\ ${ }^{2}$ Department of Applied Mathematics, School of Mathematical Sciences, Ferdowsi University of Mashhad, Mashhad, Iran \\ ${ }^{3}$ Institute for Groundwater Studies, Faculty of Natural and Agricultural Sciences, University of the Free State, \\ Bloemfontein 9300, South Africa
}

Correspondence should be addressed to S. Shateyi; stanford.shateyi@univen.ac.za

Received 26 November 2013; Accepted 12 February 2014; Published 18 March 2014

Academic Editor: Hossein Jafari

Copyright (C) 2014 Emran Tohidi et al. This is an open access article distributed under the Creative Commons Attribution License, which permits unrestricted use, distribution, and reproduction in any medium, provided the original work is properly cited.

This paper presents a computational approach for solving a class of nonlinear Volterra integro-differential equations of fractional order which is based on the Bernoulli polynomials approximation. Our method consists of reducing the main problems to the solution of algebraic equations systems by expanding the required approximate solutions as the linear combination of the Bernoulli polynomials. Several examples are given and the numerical results are shown to demonstrate the efficiency of the proposed method.

\section{Introduction}

In real world, for modeling and analysing a huge size of problems we need fractional calculus. Fractional calculus finds its application in many fields of sciences and engineering, including fluid flow, electrical networks, fractals theory, control theory, electromagnetic theory, probability, statistics, optics, potential theory, biology, chemistry, diffusion, and viscoelasticity [1-4].

In recent years, fractional differential equations (FDEs) and fractional integro-differential equations (FIDEs) have become the focus of interest for many researchers in different disciplines of science and technology because of the fact that a realistic modeling of a physical phenomenon having dependence not only on the time instant but also on the previous time history that can be successfully achieved by using fractional calculus. However, besides modeling, the solution techniques and their reliabilities are most important to catch critical points at which a sudden divergence, convergence, or bifurcation starts. Therefore, high accuracy solutions are always needed. For this purpose several techniques were proposed to solve the fractional order differential equations (or integro-differential equations). The most commonly used ideas are Adomian decomposition method (ADM) [5], variational iteration method (VIM) [6], fractional differential transform method (FDTM) [7], fractional difference method (FDM) [8], and power series method [9].

On the other hand, since the beginning of 1994, Laguerre, Legendre, Taylor, Fourier, Hermite, and Bessel (matrix and collocation) methods have been used in the works [10-15] to solve linear differential, integral, and integro-differentialdifference equations and their systems. Also, the Bernoulli (matrix and collocation) methods have been used to find the approximate solutions of differential and integro-differential equations [16-18]. To the best of our knowledge these polynomials have had no results for solving FIDEs. Moreover, according to the discussions in [18], Bernoulli polynomials have some certain properties that encourage us to use them for solving any applied mathematics problem. These subjects motivate us to present a new numerical scheme for solving FIDEs.

In this paper, by using the Bernoulli polynomials as the test functions and collocating the following FIDE (subject to sufficient initial or boundary conditions) at the Legendre 
Gauss collocation points and also approximating the existing integrals by the Gauss quadrature rule, we find the numerical solution of the following FIDE:

$$
\begin{array}{r}
D^{\alpha} y(x)=F\left(x, y(x), \int_{0}^{x} K(t, y(t)) d t\right), \\
0<x<1, \quad \alpha>0 .
\end{array}
$$

The rest of this paper is organized as follows. Some preliminaries about the fractional calculus and also the Bernoulli polynomials together with the Gauss quadrature rule are provided in the next Section. Section 3 contains the basic idea of the paper. In Section 4, several numerical examples are given to show the robustness of the proposed idea. The provided numerical examples show the efficiency of the proposed idea with regard to some methods in the literature. In the last section, we provide the conclusions.

\section{Preliminaries}

In this section, we deal with several basic definitions and properties of fractional calculus theory and also some useful information about the Bernoulli polynomials together with the Legendre Gauss quadrature rule which are further used hereafter.

Definition 1. A real function $f(x), x>0$, is said to be in the space $C_{\mu}, \mu \in \mathbb{R}$, if there exists a real number $p, p>\mu$, such that $f(x)=x^{p} f_{1}(x)$, where $f_{1}(x) \in C[0, \infty)$, and it is said to be in the space $C_{\mu}^{n}$ if and only if $f^{(n)} \in C_{\mu}, n \in \mathbb{N}_{0}=\mathbb{N} \cup\{0\}$.

Clearly, $C_{\mu}$ is a vector space and the set of spaces $C_{\mu}$ is ordered by inclusion according to

$$
C_{\mu} \subset C_{\nu} \Longleftrightarrow \mu \geq \nu .
$$

Definition 2. The Riemann-Liouville fractional integral operator of order $\alpha$ for a function in $C_{\mu}$, where $\mu \geq-1$, is defined as

$$
\begin{aligned}
& J^{\alpha} f(x)=\frac{1}{\Gamma(\alpha)} \int_{0}^{x}(x-t)^{\alpha-1} f(t) d t, \quad \alpha>0, \\
& J^{0} f(x)=f(x) .
\end{aligned}
$$

Definition 3. The fractional derivative of $f(x)$ in the Caputo sense is defined as

$$
D^{\alpha} f(x)=J^{n-\alpha} f^{(n)}(x),
$$

for $n-1<\alpha \leq n, n \in \mathbb{N}, x>0$ and $f \in C_{-1}^{n}$.

It should be mentioned that, for $\alpha \in \mathbb{N}$, the Caputo differential operator coincides with the classical differential operator of integer order. Some properties of the Caputo fractional derivative, which are needed here, are as follows:

$$
\begin{aligned}
& D^{\alpha} C=0, \quad(C \text { is a constant }) \\
& D^{\alpha} x^{\beta}=\left\{\begin{array}{l}
0, \\
\text { for } \beta \in \mathbb{N}_{0}, \beta<\lceil\alpha\rceil, \\
\frac{\Gamma(\beta+1)}{\Gamma(\beta+1-\alpha)} x^{\beta-\alpha}, \\
\text { for } \beta \in \mathbb{N}_{0}, \beta \geq\lceil\alpha\rceil \text { or } \beta \notin \mathbb{N}, \beta>\lfloor\alpha\rfloor,
\end{array}\right.
\end{aligned}
$$

where the ceiling function $\lceil\alpha\rceil$ denotes the smallest integer greater than or equal to $\alpha$ and the floor function $\lfloor\alpha\rfloor$ denotes the largest integer less than or equal to $\alpha$.

Similar to the integer order differentiation, the Caputo fractional differential operator is a linear operation; in other words

$$
\begin{array}{r}
D^{\alpha}(\theta f(x)+\lambda g(x))=\theta D^{\alpha} f(x)+\lambda D^{\alpha} g(x), \\
n-1<\alpha \leq n, \quad f, g \in C_{-1}^{n},
\end{array}
$$

where $\theta$ and $\lambda$ are constants.

Definition 4. The Bernoulli polynomials play an important role in different areas of mathematics, including number theory and the theory of finite differences. The classical Bernoulli polynomials $B_{n}(x)$ are usually defined by means of the following relations:

$$
\begin{gathered}
\frac{d B_{n}(x)}{d x}=n B_{n-1}(x), \quad(n \geq 1), \\
\int_{0}^{1} B_{n}(x) d x=0, \quad(n \geq 1), \\
B_{0}(x)=1 .
\end{gathered}
$$

Also the Bernoulli polynomials can be represented in the form

$$
B_{n}(x)=\sum_{r=0}^{N}\left(\begin{array}{l}
n \\
r
\end{array}\right) B_{n-r}(0) x^{r} .
$$

Definition 5. The Legendre Gauss quadrature rule can be defined as follows [11]:

$$
\begin{aligned}
\int_{0}^{1} h(s) d s & =\frac{1}{2} \int_{-1}^{1} h\left(\frac{1}{2}(t+1)\right) d t \\
& \approx \frac{1}{2} \sum_{i=0}^{N} w_{i} h\left(\frac{1}{2}\left(t_{i}+1\right)\right),
\end{aligned}
$$

where $t_{i}$ for $i=0,1, \ldots, N$ are the roots of the $(N+1)$ th Legendre polynomial $P_{N+1}(t)$ and $w_{i}=2 /\left(1-t_{i}^{2}\right) P_{N+1}^{\prime}\left(t_{i}\right)$.

\section{Basic Idea}

In this section, we consider the basic equation (1) with some appropriate initial or boundary conditions. Our aim is to 
approximate the solution $y(x)$ by the truncated Bernoulli series $y_{N}(x)=\sum_{n=0}^{N} c_{n} B_{n}(x)$. Moreover, we use Legendre Gauss collocation nodes and also the Legendre Gauss quadrature rule for approximating the existing integrals. Then, the basic equation (1) will be transformed to a nonlinear system of algebraic equations. The solutions of this algebraic system are $c_{0}, c_{1}, \ldots, c_{N}$. Therefore, an approximate solution of (1) will be obtained in the form $y_{N}(x)=\sum_{n=0}^{N} c_{n} B_{n}(x)$. Before presenting our main idea, we provide the Caputo fractional derivative representation of $y_{N}(x)$ in the following lemma.

Lemma 6. Let $y(x)$ be approximated by the Bernoulli polynomials as $y_{N}(x)=\sum_{n=0}^{N} c_{n} B_{n}(x)$ and also suppose that $\alpha>0$; then

$$
D^{\alpha} y_{N}(x)=\sum_{n=\lceil\alpha\rceil}^{N} \sum_{r=\lceil\alpha\rceil}^{n} c_{n} b_{n, r}^{(\alpha)} x^{r-\alpha}
$$

where $b_{n, r}^{(\alpha)}$ is given by

$$
b_{n, r}^{(\alpha)}=\frac{n !}{(n-r) ! \Gamma(r+1-\alpha)} B_{n-r}(0) .
$$

Proof. Because of the linearity of the Caputo fractional differential operator we have

$$
D^{\alpha} y_{N}(x)=\sum_{n=0}^{N} c_{n} D^{\alpha} B_{n}(x) .
$$

According to (5) and the structure of the Bernoulli polynomials,

$$
D^{\alpha} B_{n}(x)=0, \quad n=0,1, \ldots,\lceil\alpha\rceil-1, \alpha>0 .
$$

Also for $n=\lceil\alpha\rceil, \ldots, N$ and using (5) we reach the following result:

$$
\begin{aligned}
D^{\alpha} B_{n}(x) & =\sum_{r=0}^{n}\left(\begin{array}{l}
n \\
r
\end{array}\right) B_{n-r}(0) D^{\alpha} x^{r} \\
& =\sum_{r=\lceil\alpha\rceil}^{n} \frac{n !}{(n-r) ! \Gamma(r+1-\alpha)} B_{n-r}(0) x^{r-\alpha} .
\end{aligned}
$$

A combination of (12) and (14) leads to the desired result.

In this part, we turn to approximate the solution of problem (1). For this purpose, substituting approximations $y_{N}(x)$ and $D^{\alpha} y_{N}(x)$ in (1) yields

$$
\begin{aligned}
& \sum_{n=\lceil\alpha\rceil}^{N} \sum_{r=\lceil\alpha\rceil}^{n} c_{n} b_{n, r}^{(\alpha)} x^{r-\alpha} \\
& =F\left(x, \sum_{n=0}^{N} c_{n} B_{n}(x), \int_{0}^{x} K\left(t, \sum_{n=0}^{N} c_{n} B_{n}(t)\right) d t\right), \\
& 0<x<1, \quad \alpha>0 .
\end{aligned}
$$

By collocating the above equation at $(N+1-\lceil\alpha\rceil)$ points $x_{p}$ we have

$$
\begin{aligned}
& \sum_{n=\lceil\alpha\rceil}^{N} \sum_{r=\lceil\alpha\rceil}^{n} c_{n} b_{n, r}^{(\alpha)} x_{p}^{r-\alpha} \\
& =F\left(x_{p}, \sum_{n=0}^{N} c_{n} B_{n}\left(x_{p}\right), \int_{0}^{x_{p}} K\left(t, \sum_{n=0}^{N} c_{n} B_{n}(t)\right) d t\right), \\
& p=0,1, \ldots, N-\lceil\alpha\rceil,
\end{aligned}
$$

where $x_{p}$ for $p=0,1, \ldots, N-\lceil\alpha\rceil$ denote the roots of the shifted Legendre polynomial $P_{N+1-\lceil\alpha\rceil}(x)$ in the interval $[0,1]$. Also, in order to use the Legendre Gauss quadrature for approximating the abovementioned involved integrals, we should transfer $t$-interval $\left[0, x_{p}\right]$ into $\tau$-interval $[-1,1]$ by the following change of variable:

$$
\tau=\frac{2}{x_{p}} t-1
$$

For each value of $p=0,1, \ldots, N-\lceil\alpha\rceil$ the abovementioned equation may be restated as follows:

$$
\begin{aligned}
& \sum_{n=\lceil\alpha\rceil}^{N} \sum_{r=\lceil\alpha\rceil}^{n} c_{n} b_{n, r}^{(\alpha)} x_{p}^{r-\alpha} \\
&=F\left(x_{p}, \sum_{n=0}^{N} c_{n} B_{n}\left(x_{p}\right),\right. \\
&\left.\quad \frac{x_{p}}{2} \int_{-1}^{1} K\left(\frac{x_{p}}{2}(\tau+1), \sum_{n=0}^{N} c_{n} B_{n}\left(\frac{x_{p}}{2}(\tau+1)\right)\right) d \tau\right) .
\end{aligned}
$$

Therefore, applying Gaussian integration formula yields

$$
\begin{aligned}
& \sum_{n=\lceil\alpha\rceil}^{N} \sum_{r=\lceil\alpha\rceil}^{n} c_{n} b_{n, r}^{(\alpha)} x_{p}^{r-\alpha} \\
& \simeq F\left(x_{p}, \sum_{n=0}^{N} c_{n} B_{n}\left(x_{p}\right),\right. \\
& \left.\quad \frac{x_{p}}{2} \sum_{q=0}^{N} w_{q} K\left(\frac{x_{p}}{2}\left(\tau_{q}+1\right), \sum_{n=0}^{N} c_{n} B_{n}\left(\frac{x_{p}}{2}\left(\tau_{q}+1\right)\right)\right)\right),
\end{aligned}
$$

where all of the $\tau_{q}$ 's are the $N+1$ zeros of the Legendre polynomial $L_{N+1}(\tau)$ and the $w_{q}$ 's are the corresponding weights. Together with $\lceil\alpha\rceil$ equations of supplementary conditions, we get $(N+1)$ nonlinear algebraic equations which can be solved for the unknown $c_{n}, n=0,1, \ldots, N$, by using any appropriate iterative method. Consequently $y_{N}(x)$ may be obtained. In the next section, we will show the applicability of the proposed method by examining several numerical examples. 


\section{Numerical Examples}

In this section, several numerical examples are given to illustrate the accuracy and effectiveness of the proposed method, and all of them are performed on a computer using some programs written in MAPLE 13. In this regard, we have reported in the tables the values of the exact solution $y(x)$ and the polynomial approximate solution $y_{N}(x)$ at any selected points of the given interval $[0,1]$. It should be noted that, in the first example, we provide an interesting example in which our method reaches the exact solution in the polynomial form. Moreover, in the second and third examples our method reaches the same results of [19] by using lower values of approximation. Also in the last numerical example, our results are superior with respect to the CAS wavelet method [20]. Before presenting our numerical examples, we should recall that the MAPLE software for solving nonlinear system of algebraic equations suggests the fsolve command. As per our experience for solving such nonlinear systems, this command is very efficient and easy to handle.

Example 1 (see [19]). As in the first example, we consider the following initial value problem of FIDE:

$$
\begin{aligned}
D^{0.75} y(x) & =-\left(\frac{e^{x} x^{2}}{5}\right) y(x)+\frac{6 x^{2.25}}{\Gamma(3.25)}+\int_{0}^{x} e^{x} t y(t) d t \\
y(0) & =0
\end{aligned}
$$

For solving this example, we apply our proposed method for $N=3$. In other words,

$$
y(x) \approx y_{3}(x)=\sum_{n=0}^{3} c_{n} B_{n}(x) .
$$

By using (19), we have

$$
\begin{aligned}
& \sum_{n=1}^{3} \sum_{r=1}^{n} c_{n} b_{n, r}^{(0.75)} x_{p}^{r-0.75} \\
& \simeq-\left(\frac{e^{x_{p}} x_{p}^{2}}{5}\right) \sum_{n=0}^{3} c_{n} B_{n}\left(x_{p}\right)+\frac{6 x_{p}^{2.25}}{\Gamma(3.25)} \\
& \quad+\frac{x_{p}}{2} e^{x_{p}} \sum_{q=0}^{3} w_{q}\left(\frac{x_{p}}{2}\left(\tau_{q}+1\right) \sum_{n=0}^{3} c_{n} B_{n}\left(\frac{x_{p}}{2}\left(\tau_{q}+1\right)\right)\right), \\
& p=0,1,2 .
\end{aligned}
$$

Initial condition yields the following equation:

$$
c_{0}-\frac{1}{2} c_{1}+\frac{1}{6} c_{2}=0
$$

Now, by solving the system which contains (22) and (23) we reach the following solution:

$$
c_{0}=\frac{1}{4}, \quad c_{1}=1, \quad c_{2}=\frac{3}{2}, \quad c_{3}=1 .
$$

Therefore,

$$
\begin{aligned}
y_{3}(x)= & \frac{1}{4}(1)+1\left(x-\frac{1}{2}\right)+\frac{3}{2}\left(x^{2}-x+\frac{1}{6}\right) \\
& +1\left(x^{3}-\frac{3}{2} x^{2}+\frac{1}{2} x\right)=x^{3}
\end{aligned}
$$

which is the exact solution.

Example 2 (see [19]). As in the second example, we consider the following fractional integro-differential equation:

$$
\begin{array}{r}
D^{\alpha} y(x)=x\left(1+e^{x}\right)+3 e^{x}+y(x)-\int_{0}^{x} y(t) d t \\
0<x<1, \quad 3<\alpha \leq 4,
\end{array}
$$

with the following boundary conditions:

$$
\begin{aligned}
y(0) & =1, \quad y^{\prime \prime}(0)=2, \quad y(1)=1+e, \\
y^{\prime \prime}(1) & =3 e .
\end{aligned}
$$

The exact solution of this FIDE is $y(x)=1+x e^{x}$, when $\alpha=4$. For solving this problem, we apply our method for different values of $N$. In Table 1 , we provide the numerical results $y_{N}\left(x_{i}\right)$, where $x_{i}=i / 10(i=0,1, \ldots, 10)$ for $N=8$, of our presented method (PM) together with numerical results $y_{N}\left(x_{i}\right)$, where $x_{i}=i / 10$ for $N=10$, of the Legendre collocation method (LCM) [19]. It is obvious that our method reaches the same results of [19] with lower degree of approximation. Moreover, our method has superior results with regard to the Adomian decomposition method (ADM) [5] as shown in [19]. In addition, the numerical results associated with our presented method, LCM, and generalized differential transform method (GDTM) [7] for $N=10$ and $\alpha=3.75$ are given in Table 2. As shown in Table 2 of [19], the ADM has very weak approximations with regard to GDTM and LCM. Therefore, we do not consider ADM in Table 2. From this table, one can find that our results are the same as those of LCM, but GDTM results are away from our proposed technique and LCM results. These facts confirm the effectiveness of our idea. For showing the reliability of our approach, we provide Figure 1. In this figure, we depict the numerical solution $y_{10}(x)$ for different kinds of $\alpha$ such as 3.25, $3.50,3.75$, and 4 .

Example 3 (see [19]). As in the third example, we consider the following nonlinear fractional integro-differential equation:

$$
D^{\alpha} y(x)=1+\int_{0}^{x} e^{-t} y^{2}(t) d t, \quad 0<x<1,3<\alpha \leq 4,
$$

subject to the boundary conditions

$$
\begin{aligned}
y(0) & =1, \quad y^{\prime \prime}(0)=1, \quad y(1)=e, \\
y^{\prime \prime}(1) & =e .
\end{aligned}
$$




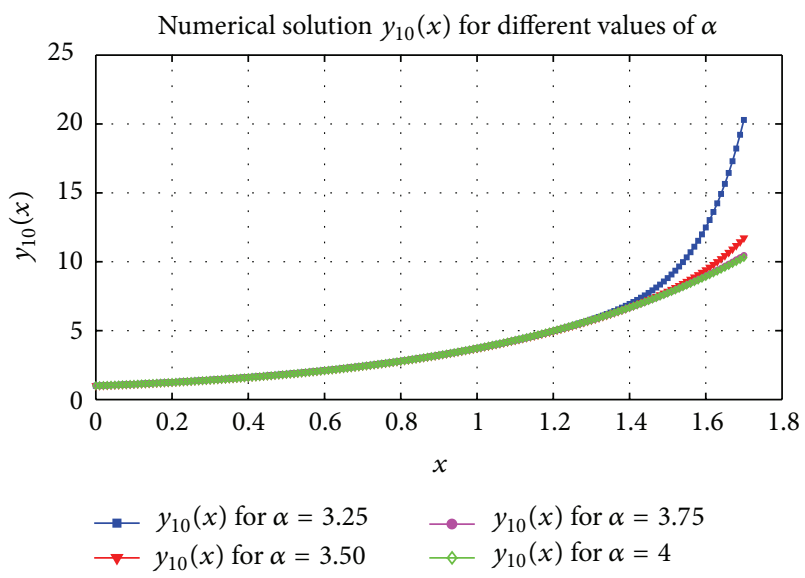

Figure 1: Numerical solution history of Example 2 for $\alpha=3.25$, $3.50,3.75$, and 4 .

TABLE 1: Numerical results of Example 2 for $\alpha=4$.

\begin{tabular}{cccc}
\hline$x_{i}$ & LCM for $N=10$ & $\begin{array}{c}\alpha=4 \\
\text { PM for } N=8\end{array}$ & Exact solution \\
\hline 0.0 & 1.00000000 & 1.00000000 & 1.00000000 \\
0.1 & 1.11051709 & 1.11051709 & 1.11051709 \\
0.2 & 1.24428055 & 1.24428055 & 1.24428055 \\
0.3 & 1.40495764 & 1.40495765 & 1.40495764 \\
0.4 & 1.59672988 & 1.59672989 & 1.59672988 \\
0.5 & 1.82436064 & 1.82436063 & 1.82436064 \\
0.6 & 2.09327128 & 2.09327126 & 2.09327128 \\
0.7 & 2.40962690 & 2.40962687 & 2.40962690 \\
0.8 & 2.78043274 & 2.78043273 & 2.78043274 \\
0.9 & 3.21364280 & 3.21364280 & 3.21364280 \\
1.0 & 3.71828183 & 3.71828183 & 3.71828183 \\
\hline
\end{tabular}

TABLE 2: Numerical results of Example 2 for $\alpha=3.75$.

\begin{tabular}{cccc}
\hline$x_{i}$ & \multicolumn{3}{c}{$\alpha=3.75$} \\
& LCM for $N=10$ & PM for $N=10$ & GDTM for $N=10$ \\
\hline 0.0 & 1.00000000 & 1.00000000 & 1.00000000 \\
0.1 & 1.11580022 & 1.11580022 & 1.11576401 \\
0.2 & 1.25417406 & 1.25417406 & 1.25411023 \\
0.3 & 1.41835392 & 1.41835392 & 1.41826880 \\
0.4 & 1.61225031 & 1.61225031 & 1.61215425 \\
0.5 & 1.84049469 & 1.84049469 & 1.84039953 \\
0.6 & 2.10850149 & 2.10850149 & 2.10841524 \\
0.7 & 2.42253768 & 2.42253768 & 2.42246558 \\
0.8 & 2.78981125 & 2.78981125 & 2.78975920 \\
0.9 & 3.21858158 & 3.21858158 & 3.21855471 \\
1.0 & 3.71828183 & 3.71828183 & 3.71828183 \\
\hline
\end{tabular}

The exact solution of this problem for $\alpha=4$, that is, the classical boundary value problem, is $y(x)=e^{x}$. Similar to the previous example, for solving this example, we apply our presented technique for different values of $N$. In Table 3 , we provide the numerical results $y_{N}\left(x_{i}\right)$, where $x_{i}=i / 10$
TABLE 3: Numerical results of Example 3 for $\alpha=4$ and $\alpha=3.75$.

\begin{tabular}{cccc}
\hline$x_{i}$ & \multicolumn{3}{c}{$\alpha=3.75$} \\
& LCM for $N=13$ & PM for $N=10$ & GDTM for $N=13$ \\
\hline 0.0 & 1.00000000 & 1.00000000 & 1.00000000 \\
0.1 & 1.10618147 & 1.10618610 & 1.10617705 \\
0.2 & 1.22328484 & 1.22329275 & 1.22327680 \\
0.3 & 1.35238877 & 1.35239964 & 1.35237837 \\
0.4 & 1.49473312 & 1.49474546 & 1.49472145 \\
0.5 & 1.65172131 & 1.65173324 & 1.65170946 \\
0.6 & 1.82492929 & 1.82494001 & 1.82491846 \\
0.7 & 2.01611828 & 2.01612731 & 2.01610929 \\
0.8 & 2.22724889 & 2.22725546 & 2.22714245 \\
0.9 & 2.46049864 & 2.46050202 & 2.46049530 \\
1.0 & 2.71828183 & 2.71828183 & 2.71828183 \\
\hline$x_{i}$ & & $\alpha=4$ & \\
\hline 0.0 & 1.00000000 & 1.00000000 & 1.00000000 \\
0.1 & 1.10517092 & 1.10517092 & 1.10517092 \\
0.2 & 1.22140276 & 1.22140276 & 1.22140276 \\
0.3 & 1.34985881 & 1.34985881 & 1.34985881 \\
0.4 & 1.49182470 & 1.49182470 & 1.49182470 \\
0.5 & 1.64872127 & 1.64872127 & 1.64872127 \\
0.6 & 1.82211880 & 1.82211880 & 1.82211880 \\
0.7 & 2.01375271 & 2.01375271 & 2.01375271 \\
0.8 & 2.22554093 & 2.22554093 & 2.22554093 \\
0.9 & 2.45960311 & 2.45960311 & 2.45960311 \\
1.0 & 2.71828183 & 2.71828183 & 2.71828183 \\
\hline & & & \\
\hline
\end{tabular}

$(i=0,1, \ldots, 10)$ for $N=9$, of our presented method (PM) together with numerical results $y_{N}\left(x_{i}\right)$, where $x_{i}=i / 10$ for $N=13$, of the LCM in the case of $\alpha=4$. From this table, it is obvious that our method reaches the same results of [19] with lower degree of approximation. Moreover, in this table the numerical results $y_{N}\left(x_{i}\right)$, where $x_{i}=i / 10(i=0,1, \ldots, 10)$ for $N=10$, of our presented method (PM) together with the numerical results $y_{N}\left(x_{i}\right)$, where $x_{i}=i / 10$ for $N=13$, of the LCM and GDTM in the case of $\alpha=3.75$ are given.

Example 4 (see [20]). As in the final example, let us consider the following nonlinear fractional integro-differential equation of order $\alpha=6 / 5$ :

$$
\begin{array}{r}
D^{\alpha} y(x)=\frac{5}{2 \Gamma(4 / 5)} x^{4 / 5}-\frac{x^{9}}{252}+\int_{0}^{x}(x-t)^{2} y^{3}(t) d t \\
0 \leq x<1,
\end{array}
$$

with the supplementary conditions

$$
y(0)=0, \quad y(1)=1 .
$$

The exact solution of this problem is $y(x)=x^{2}$. Again, we solve this problem by using our basic idea in Section 3 . For making a real comparison with a new technique, CAS wavelet method (CASWM) [20], we should assume that $N=$ 
TABLE 4: Numerical results of Example 4 for $\alpha=6 / 5$.

\begin{tabular}{cccc}
\hline \multicolumn{4}{c}{$\alpha=6 / 5$} \\
$x_{i}$ & CASWM for $k=2, M=1$ & PM for $N=2$ & Exact solution \\
\hline 0.0 & 0.04 & $1.0 e-012$ & 0.00000000 \\
0.1 & 0.02 & 0.00992974 & 0.01 \\
0.2 & 0.05 & 0.03987509 & 0.04 \\
0.3 & 0.12 & 0.08983606 & 0.09 \\
0.4 & 0.18 & 0.15981264 & 0.16 \\
0.5 & 0.32 & 0.24980483 & 0.25 \\
0.6 & 0.41 & 0.35981264 & 0.36 \\
0.7 & 0.54 & 0.48983606 & 0.49 \\
0.8 & 0.68 & 0.63987509 & 0.64 \\
0.9 & 0.89 & 0.80992974 & 0.81 \\
1.0 & 0.90 & 1.00000000 & 1 \\
\hline
\end{tabular}

2 and then solve the abovementioned problem. Therefore, in Table 4, we provide the numerical results $y_{N}\left(x_{i}\right)$, where $x_{i}=i / 10(i=0,1, \ldots, 10)$ for $N=2$, of our presented method (PM) together with the numerical results $y_{N}\left(x_{i}\right)$, where $x_{i}=i / 10$ for $N=2$, of the CASWM. From this table the efficiency of the presented method could be seen obviously.

\section{Conclusions}

In this paper, the Bernoulli polynomials and Legendre Gauss quadrature rule together with the Legendre Gauss collocation nodes are used to reduce the nonlinear fractional integrodifferential equations with appropriate initial or boundary conditions to the solution of system of nonlinear algebraic equations. From the computational point of view, the solution obtained by this method is in excellent agreement with those obtained by previous works and also it is efficient to use. One issue of future work is to develop a similar technique to solve some interesting nonlinear fractional partial integrodifferential equations. In addition, the method can also be extended to the system of nonlinear fractional integrodifferential equations, but some modifications are required.

\section{Conflict of Interests}

The authors declare that they do not have any conflict of interests in their submitted paper.

\section{References}

[1] A. E. M. El-Mesiry, A. M. A. El-Sayed, and H. A. A. ElSaka, "Numerical methods for multi-term fractional (arbitrary) orders differential equations," Applied Mathematics and Computation, vol. 160, no. 3, pp. 683-699, 2005.

[2] A. A. Kilbas, H. M. Srivastava, and J. J. Trujillo, Theory and Applications of Fractional Differential Equations, Elsevier, New York, NY, USA, 2006.

[3] K. S. Miller and B. Ross, An Introduction to the Fractional Calculus and Fractional Differential Equations, John Wiley \& Sons, New York, NY, USA, 1993.
[4] I. Podlubny, Fractional Differential Equations, Academic Press, New York, NY, USA, 1999.

[5] S. A. El-Wakil, A. Elhanbaly, and M. A. Abdou, "Adomian decomposition method for solving fractional nonlinear differential equations," Applied Mathematics and Computation, vol. 182, no. 1, pp. 313-324, 2006.

[6] S. Das, "Analytical solution of a fractional diffusion equation by variational iteration method," Computers \& Mathematics with Applications, vol. 57, no. 3, pp. 483-487, 2009.

[7] V. S. Erturk, S. Momani, and Z. Odibat, "Application of generalized differential transform method to multi-order fractional differential equations," Communications in Nonlinear Science and Numerical Simulation, vol. 13, no. 8, pp. 1642-1654, 2008.

[8] M. M. Meerschaert and C. Tadjeran, "Finite difference approximations for two-sided space-fractional partial differential equations," Applied Numerical Mathematics, vol. 56, no. 1, pp. 80-90, 2006.

[9] Z. M. Odibat and N. T. Shawagfeh, "Generalized Taylor's formula," Applied Mathematics and Computation, vol. 186, no. 1, pp. 286-293, 2007.

[10] M. Gülsu, B. Gürbüz, Y. Öztürk, and M. Sezer, "Laguerre polynomial approach for solving linear delay difference equations," Applied Mathematics and Computation, vol. 217, no. 15, pp. 6765-6776, 2011.

[11] O. R. N. Samadi and E. Tohidi, “The spectral method for solving systems of Volterra integral equations," Journal of Applied Mathematics and Computing, vol. 40, no. 1-2, pp. 477-497, 2012.

[12] E. Tohidi, F. Soleymani, and A. Kilicman, "Robustness of operational matrices of differentiation for solving state-space analysis and optimal control problems," Abstract and Applied Analysis, vol. 2013, Article ID 535979, 9 pages, 2013.

[13] F. Toutounian, E. Tohidi, and A. Kilicman, "Fourier operational matrices of differentiation and transmission: introduction and applications," Abstract and Applied Analysis, vol. 2013, Article ID 198926, 11 pages, 2013.

[14] S. Yalçinbaş, M. Aynigül, and M. Sezer, "A collocation method using Hermite polynomials for approximate solution of pantograph equations," Journal of the Franklin Institute, vol. 348, no. 6, pp. 1128-1139, 2011.

[15] S. Yüzbaş1, "A numerical approximation based on the Bessel functions of first kind for solutions of Riccati type differentialdifference equations," Computers \& Mathematics with Applications, vol. 64, no. 6, pp. 1691-1705, 2012.

[16] E. Tohidi and A. Kılıçman, "A collocation method based on the Bernoulli operational matrix for solving nonlinear BVPs which arise from the problems in calculus of variation," Mathematical Problems in Engineering, vol. 2013, Article ID 757206, 9 pages, 2013.

[17] E. Tohidi, Kh. Erfani, M. Gachpazan, and S. Shateyi, "A new Tau method for solving nonlinear Lane-Emden type equations via Bernoulli operational matrix of differentiation," Journal of Applied Mathematics, vol. 2013, Article ID 850170, 9 pages, 2013.

[18] F. Toutounian, E. Tohidi, and S. Shateyi, "A collocation method based on the Bernoulli operational matrix for solving highorder linear complex differential equations in a rectangular domain," Abstract and Applied Analysis, vol. 2013, Article ID 823098, 12 pages, 2013. 
[19] A. Saadatmandi and M. Dehghan, "A Legendre collocation method for fractional integro-differential equations," Journal of Vibration and Control, vol. 17, no. 13, pp. 2050-2058, 2011.

[20] H. Saeedi and M. M. Moghadam, "Numerical solution of nonlinear Volterra integro-differential equations of arbitrary order by CAS wavelets," Communications in Nonlinear Science and Numerical Simulation, vol. 16, no. 3, pp. 1216-1226, 2011. 


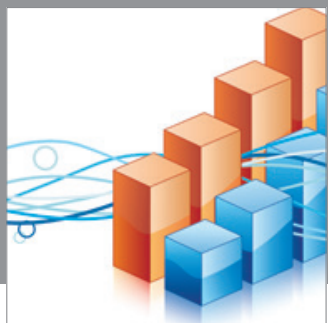

Advances in

Operations Research

mansans

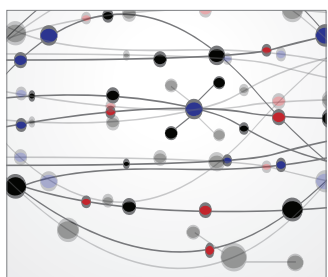

The Scientific World Journal
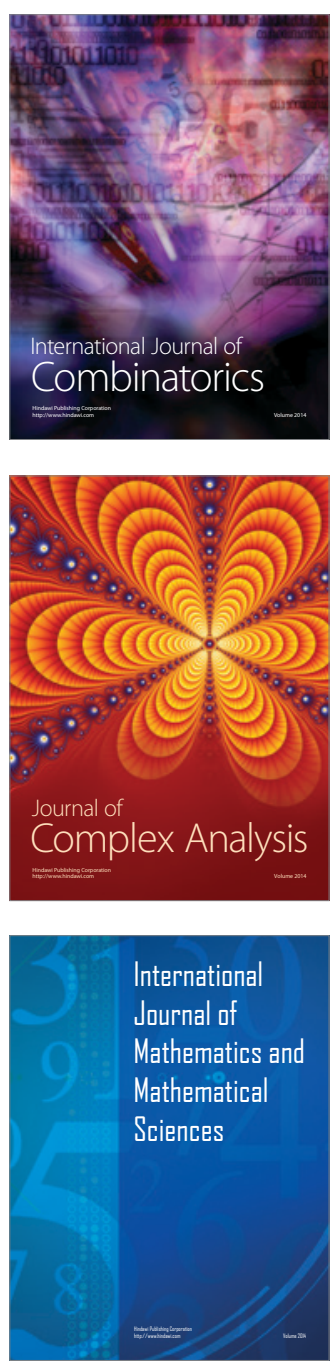
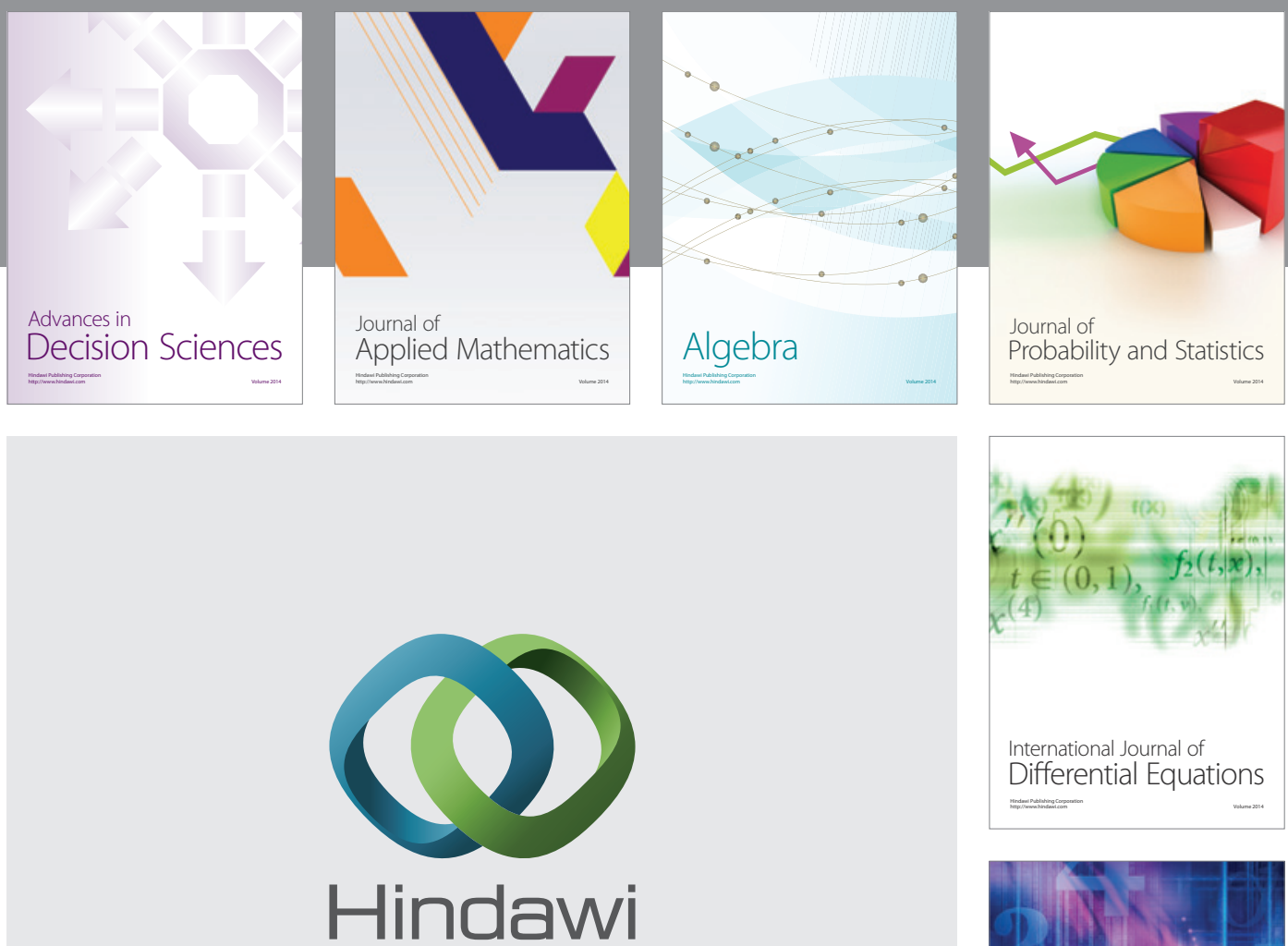

Submit your manuscripts at http://www.hindawi.com
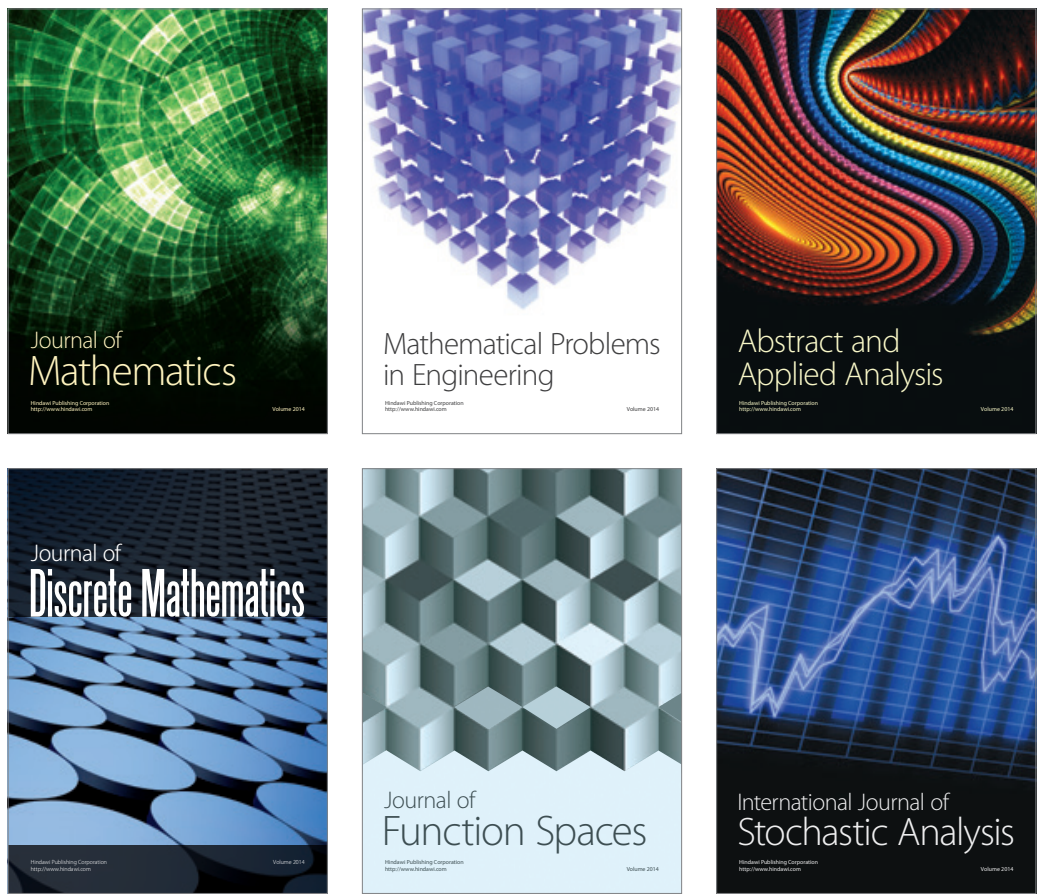

Journal of

Function Spaces

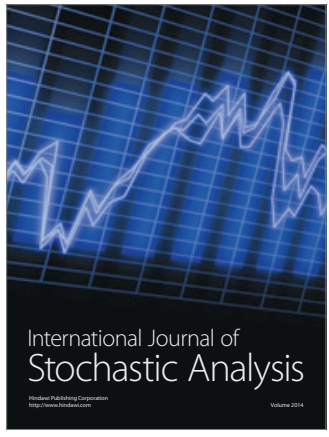

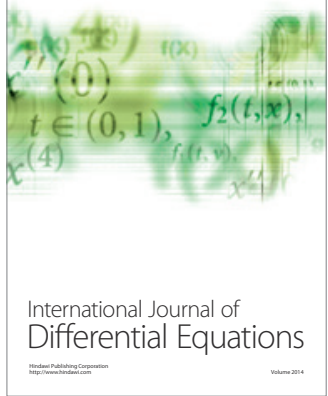
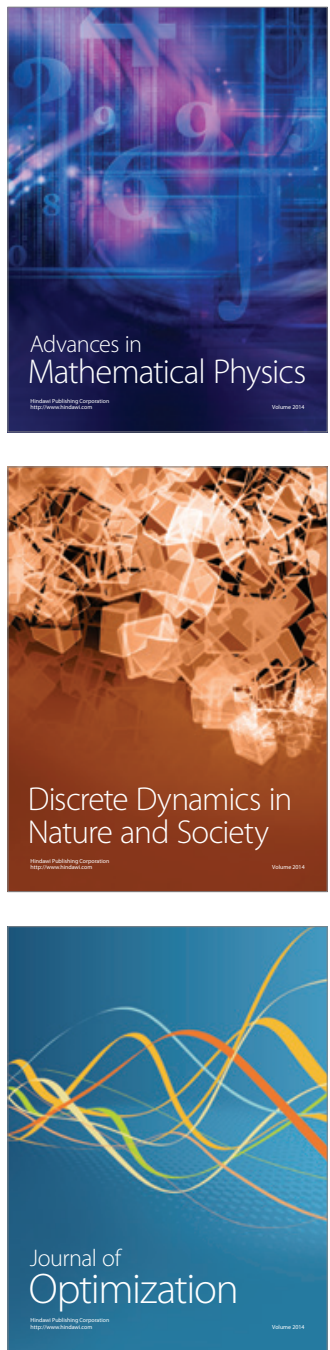